\title{
SCIDioc
}

\author{
International Journal of Dentistry and Oral Science (IJDOS) \\ ISSN: 2377-8075
}

\section{Geometric Morphometric Analysis (GMA) Of Mandibular Symphysis In Class Iii Skeletal Base Patients}

Research Article

\author{
Murshida Marizan Nor $^{1 *}$, Alizae Marny Mohamed ${ }^{1}$, Helmi Mohd Hadi Pritam², Teo Kui Fei ${ }^{3}$ \\ ${ }^{1}$ Senior lecturer, Orthodontic Discipline, Department of Family Oral Health, Faculty of Dentistry, Universiti Kebangsaan Malaysia, Jalan Raja Muda \\ Abdul Aziz, 50300 Kuala Lumpur, Malaysia. \\ ${ }^{1}$ Senior lecturer, Department of Family Oral Health, Faculty of Dentistry, Universiti Kebangsaan Malaysia, Jalan Raja Muda Abdul Aziz, 50300 Kuala \\ Lumpur, Malaysia. \\ ${ }^{2}$ Senior Lecturer, School of Health Sciences, Universiti Sains Malaysia, Kubang Kerian, 16150 Kota Bharu, Kelantan. \\ ${ }^{3}$ Postgraduate Student, Department of Family Oral Health, Faculty of Dentistry, Universiti Kebangsaan Malaysia, Jalan Raja Muda Abdul Aziz, 50300 \\ Kuala Lumpur, Malaysia.
}

\section{Abstract}

Objective: To investigate the mandibular symphysis (MS) shape variation among adolescents, emerging adults, and adults with Class III skeletal base, using geometric morphometric analysis.

Materials and Methods: Pre-treatment lateral cephalometric radiographs of 254 patients aged between 11-40 years old, with Class III skeletal base $\left(\mathrm{ANB}<1^{\circ}\right.$ ) and lower incisor angle $\left(\mathrm{LIA}<99^{\circ}\right)$ were included. The sample was divided in to adolescents, emerging adults, and adults based on gonial angle $\left(>130^{\circ}\right.$ : high angle, $120^{\circ}-130^{\circ}$ : average angle and $<120^{\circ}$ : low angle). Nine landmarks with $\mathrm{x}$ and y coordinates were identified on MS. Shape and statistical analysis carried out using Morpho J version 1.06d (Klingenberg Lab, Manchester, UK).

Results: In high gonial angle group, adults had significantly narrower alveolar and basal part of MS $(\mathrm{p}=0.003)$ while emerging adults had significantly narrower alveolar part of MS $(\mathrm{p}=0.01)$ compared to adolescents. In average gonial angle group, only adults had a significantly narrower alveolar and basal part of MS compared to emerging adults $(\mathrm{p}=0.007)$ and adolescents $(p<0.0001)$. Low gonial angle group showed wider and shorter MS with no significant shape variation for all age groups. Multivariate regression showed significant shape variation of MS shape with increasing age $(p<0.0001)$.

Conclusion: The shape of the MS can be affected by age groups where significant MS shape variation observed between adolescents, emerging adults, and adults. Hence, understanding the shape variation of MS especially in adults with high gonial angle is important during orthodontic treatment planning to avoid unwanted complications.

Keywords: Geometric Morphometric Analysis; Mandibular Symphysis; Age; Class Iii.

\section{Introduction}

Mandibular symphysis (MS) is the junction where two halves of the mandible fused. It is part of the mandible in which the lower incisors and anterior portion of the chin are located. The suture of MS ossifies at 6-9 months after birth and is known to be a stable structure [1]. Longitudinal study revealed that there was an increase in the anteroposterior dimension of MS from age 8-17 years old [2]. Bone resorption of the MS takes place at the labial alveolar process and bone deposition occurs at the lingual side of the symphysis [3, 4]. A study of MS in growing children using Bjork superimposition found a significant lingual movement of B-point when increasing age [5] Previously, the growth of the mandible was known to cease at the age of 21-22 years old [6].

*Corresponding Author:

Murshida Marizan Nor,

Senior Lecturer, Orthodontic Discipline, Department of Family Oral Health, Faculty of Dentistry, Universiti Kebangsaan Malaysia, Jalan Raja Muda Abdul Aziz, 50300 Kuala Lumpur, Malaysia.

Email Id: murshida@ukm.edu.my

Received: April 27, 2021

Accepted: June 11, 2021

Published: June 13, 2021

Citation: Murshida Marizan Nor, Alizae Marny Mohamed, Helmi Mohd Hadi Pritam, Teo Kui Fei. Geometric Morphometric Analysis (GMA) Of Mandibular Symphysis In Class Iii Skeletal Base Patients. Int J Dentistry Oral Sci. 2021;08(06):2716-2720.

Copyright: Murshida Marizan Nor 2021 . This is an open-access article distributed under the terms of the Creative Commons Attribution License, which permits unrestricted use, distribution and reproduction in any medium, provided the original author and source are credited. 
However, study by Rofl Behrent found that even though the rate of change decreases with age, changes in craniofacial skeletal structure does not stop at adult age [7].

Previous studies on MS were carried out using conventional linear and angular measurements which had limitations in defining the round and curved shape of MS. Size is usually the confounder [8] and makes statistical interpretation problematic. It has also been shown that conventional cephalometric studies which are based on angular and linear measurement were insufficient to analyze shape changes of complex craniofacial anatomical forms [9]. With the limitation mentioned, geometric morphometric analysis becomes useful.

Geometric Morphometric Analysis (GMA) has become well known recently due to its ability to measure the shape of objects and provide a graphical representation of statistical interpretation. It is a tool that uses cartesian landmark coordinates to study the shape variation of an object. Since GMA studies the shape of an object, it has the upper hand in the study of morphological variation, craniofacial growth, the association between morphology and various conditions as well as treatment outcomes. It has recently gained popularity in dentistry especially in the field of Orthodontics as it is concerned with changes of shape and size of the face and its components, particularly craniofacial growth. This technique can be beneficial in the measurement of all facial and cranium structures, as well as proportions and relations between anatomical components. In addition, the size of a structure would not be a cofounder in statistical analysis as GMA analysis is based on shape coordinates after separating shape from the overall size, position, and orientation of the landmark configurations [10].

The understanding of MS morphology is important during treatment planning as it provides essential information in determining the limits of orthodontic proclination or retroclination of the lower incisors. For adult patients with skeletal Class III, orthodontic camouflage involves retroclination of lower incisors. This natural compensation may be enhanced to achieve a class I incisor relationship by using smaller rectangular or round stainless-steel archwires to facilitate lingual tipping of the mandibular incisors. Conversely, in patients with severe Class III skeletal discrepancies, significant decompensation of the lower incisors is usually required to facilitate orthognathic surgery. This is achieved by uprighting or proclining the lower incisors within the thin alveolus. Presence of crowding and thin biotype can further complicate this process by pushing the teeth beyond its bony envelope and result in gingival recession. Therefore, the movement of lower incisors is limited by the width of MS. Excessive orthodontic tooth movement may place lower incisors outside the envelope of MS or cortical plate and result in gingival recession, severe root resorption and bony dehiscence [11]. Limiting lower incisor movement within the MS during orthodontic treatment is thought to be paramount for achieving better results, stability, and periodontal health [12].

This study aimed to find out whether the shape of MS varies from adolescents to adults of Class III sekeletal base and how much bone changes can occur in this area as age increases. To overcome the limitation in defining the shape of MS, geometric morphometric analysis was used to compare the shape variation of MS in adolescents and adults with class III skeletal bases.

\section{Materials and Methods}

Ethical approval was obtained from the Ethics Committee of Medical Centre UKM (UKM PPI/111/8/JEP-2018-509). This was a cross-sectional study where lateral cephalometric radiograph (LCR) was taken on patients who seek orthodontic treatment in Faculty of Dentistry, Universiti Kebangsaan Malaysia (UKM) from 2010-2019. The inclusion criteria were patients aged 11-40 years old with class III skeletal with $\mathrm{ANB}<1^{\circ}$, LIA $<99^{\circ}$, no history of previous orthodontic treatment and no significant medical history nor taking bisphosphonates. We excluded patients with missing lower incisors, history of periodontitis, dental anomalies, syndromic and craniofacial deformities as well as poor image quality of the lateral cephalograms. Finally, a total of 254 LCR were included in this study.

All LCR were obtained using Planmeca Romexis software (Planmeca ProMax 3D Classic) following these exposure parameters: 60-90 kV, 1-14 mA, exposure time between $9-37 \mathrm{~s}$ and a sourcemidsagittal plane distance of $1.5 \mathrm{~m}$. The LCR were digitally traced using VistaDent OC(2D) (GAC International, Inc., Bohemia, NY, USA). The subjects were first classified based on gonial angle: high angle $\left(>130^{\circ}\right)$, average angle $\left(120-130^{\circ}\right)$ and low angle $\left(<120^{\circ}\right) .13$ The gonial angle were measured from the inclination of the posterior border of ramus and the inferior border of the horizontal body of the mandible [13]. The samples were further divided into adolescence (11-18 years old), emerging adults $(>18$ 24 years old) and adults ( $\geq 25$ years old) based on the patient's age at the time the radiograph was taken [14].

\section{Landmark Placement}

Nine fixed landmarks were placed on MS using TPSDig2 software [15] to comprehensively capture the shape of MS (Table 1, Figure 1). As most landmarks on MS were type III landmarks, the Frankfort horizontal plane was used to define the precise location of all landmarks. Type III landmark is defined as points along a curve or surface, in relation to some other more distant structure [16].

\section{Shape Analysis and Statistical Analysis}

TPSDig2 version 2.30 [15] generated $\mathrm{x}$ and $\mathrm{y}$ coordinates of each landmark which were then exported into Morpho J version 1.06d (Klingenberg Lab, Manchester, UK) for shape analysis. Procrustes superimposition was performed where all samples were translated, scaled and rotated to remove irrelevant information such as size, orientation and position for shape comparison. Procrustes ANOVA assessed the effect of age groups on MS variation. Canonical variate analysis (CVA) assessed the shape differences among age groups. Multivariate regression assessed the correlation of MS shape changes with increasing age. P-value was calculated based on 10,000 permutations. Alpha level for all statistical analyses was set at 0.05 .

\section{Measurement Error}

For the accuracy of LCR tracing and landmark placement, both intra-examiner and inter-examiner reliability tests were carried out on 26 randomly selected LCR at 2 weeks apart using intraclass correlation coefficients test (ICC). Both intra and inter-reliability tests were above 0.95 and above 0.89 , respectively. ICCs for land- 
mark placement on MS showed a high degree of reliability for repeated measurement with 0.999 for the x coordinates and 0.987 for the y coordinates.

\section{Results}

A total of 254 LCR (87 adolescents, 94 emerging adults and 73 adults) with mean age of $21.78 \pm 5.95$ years old were included in this study (Table 2). All subjects presented with mean ANB and LIA values of $-2.44 \pm 2.31$ and $85.58+/-7.63$, respectively.

\section{Procrustes ANOVA}

Procrustes ANOVA assessed the effect of age groups on MS size and shape variation. There was a significant difference in the shape $(p<0.0001)$ and centroid size $(p=0.002)$ of the MS with different age groups (Table 3).

\section{Canonical Variate Analysis (CVA)}

Canonical variate (CV) scores in high angle gonial group showed a gradient of narrow to wide MS especially at B-B0 point along CV1, with a marked concentration of adult MS at low CV1 scores $(-2$ to +0.5$)$. For emerging adults, the MS was concentrated at the negative end of CV1 $(-2.0$ to +1.0$)$ which was similar to adults, but its distribution dispersed from positive to negative end of CV2 (-1.5 to +2.5). The distribution of adolescents' MS had a wider dispersion across CV1 (-2.0 to 3.5) with more at the positive end of CV1. Significant shape variation observed between adolescence with adults $(\mathrm{p}=0.003)$ and emerging adults $(\mathrm{p}=0.01)$. This indicates adolescents have a wider and thicker alveolar and basal part of MS compared to adults and emerging adults (Figure 2, Table 4).

In the average gonial angle group, adults have a significantly narrower alveolar and basal part of MS compared to emerging adults $(\mathrm{p}=0.007)$ and adolescents $(\mathrm{p}<0.0001)$. Adults' MS was concentrated at the positive end of CV1 ( -1 to +3$)$ and mid-range of CV2 (-1.5 to 1.5) indicating a narrower and more constricted alveolar part, elongated and slim basal part of the MS. Both emerging adults and adolescents' scatter points were mainly concentrated at the middle to negative end of CV1 (-3.0 to 2.0) and CV2 (-2.0 to 3.0) showing a wider, and bulbous MS compared to adults. Nevertheless, no significant shape variation observed between adolescents and emerging adults ( $>00.05)$ (Figure 3, Table 4).

In low gonial angle group, the gradient of shape change along the $\mathrm{CV} 1$ is very minimal where the basal part is very similar from positive end to negative end. The alveolar and basal part of MS

Table 1. Description of landmarks on mandibular symphysis.

\begin{tabular}{|c|c|c|c|}
\hline No & Type of landmark & Name & Description \\
\hline 1 & Type II & Labial alveolar crest & The highest point of the labial alveolar crest \\
\hline 2 & Type III & B-point & $\begin{array}{c}\text { The deepest point on the curved profile of the mandible between the } \\
\text { chin and alveolar crest }\end{array}$ \\
\hline 3 & Type III & Pogonion & The most anterior point on the bony chin \\
\hline 4 & Type III & Menton & The most inferior point of the MS in the midline \\
\hline 5 & Type III & The most anterior and inferior point on the bony chin \\
\hline 6 & Type III & The most posterior point of bony chin \\
\hline 7 & Type III & The most posterior and inferior point on the bony chin \\
\hline 8 & Type III & Borpoint & Lingual to B-point with the reference to long axis of lower incisor \\
\hline 9 & Type II & $\begin{array}{c}\text { Lingual alveolar } \\
\text { crest }\end{array}$ & The highest point of the lingual alveolar crest \\
\hline
\end{tabular}

Table 2. Demographic information.

\begin{tabular}{|c|c|c|c|c|c|}
\hline \multirow{2}{*}{\multicolumn{2}{|c|}{ Variables }} & \multicolumn{3}{|c|}{ Age groups } & \multirow{2}{*}{$\begin{array}{c}\text { Total } \\
\mathrm{N}=254\end{array}$} \\
\hline & & $\begin{array}{c}\text { Adolescence } \\
(n=87)\end{array}$ & $\begin{array}{l}\text { Emerging Adulthood } \\
(\mathrm{n}=94)\end{array}$ & $\begin{array}{c}\text { Adulthood } \\
(n=73)\end{array}$ & \\
\hline \multicolumn{2}{|c|}{ Age (Mean+/-SD) } & $15.79 \pm 1.96$ & $21.57 \pm 1.58$ & $29.18 \pm 4.22$ & \\
\hline \multirow{3}{*}{$\begin{array}{c}\text { Gonial } \\
\text { angle, } \mathrm{n} \\
(\%)\end{array}$} & High & $32(42.7)$ & $24(32.0)$ & $19(25.3)$ & 75 \\
\hline & Average & $41(35.3)$ & $42(36.2)$ & $33(28.4)$ & 116 \\
\hline & low & $14(22.2)$ & $28(44.4)$ & $21(33.3)$ & 63 \\
\hline
\end{tabular}

Table 3. The centroid size and shape.

\begin{tabular}{|c|c|c|c|c|c|}
\hline Effect & SS & MS & df & F & P \\
\hline Shape & 0.060 & 0.002 & 28 & 3.110 & $<0.0001^{* \neq}$ \\
\hline Centroid size & 2.664 & 1.332 & 2 & 6.210 & $0.002^{* \neq}$ \\
\hline
\end{tabular}

SS, Sum of squares, MS, Mean squares, df, degree of freedom and F, Goodall's F statistics, " $\mathrm{p}<0.05$, FProcrustes Anova performed. 
Table 4. Pairwise comparisons of mandibular shape.

\begin{tabular}{|c|c|c|c|}
\hline Group & Age group & Age group & $\mathbf{P}$ \\
\hline \multirow{3}{*}{ High } & \multirow{2}{*}{ Adolescents } & Emerging adults & $0.0110^{*} \neq$ \\
\hline & & Adults & $0.0031^{* \neq}$ \\
\hline & Emerging adults & Adults & $0.7315^{\neq}$ \\
\hline \multirow{3}{*}{$\begin{array}{c}\text { Aver- } \\
\text { age }\end{array}$} & \multirow{2}{*}{ Adolescents } & Emerging adults & $0.0631^{\neq}$ \\
\hline & & Adults & $<0.0001^{* \neq}$ \\
\hline & Emerging adults & Adults & $0.0072^{* \neq}$ \\
\hline \multirow{3}{*}{ Low } & \multirow{2}{*}{ Adolescents } & Emerging adults & $0.0953^{\neq}$ \\
\hline & & Adults & $0.3341^{\neq}$ \\
\hline & Emerging adults & Adults & $0.0089^{*} \neq$ \\
\hline
\end{tabular}

are rounder, bulbous and shorter in all age groups compared to high and average gonial angle groups. Adolescents' MS was concentrated at the positive end of CV2 (-1.0 to +2.5) with wider MS compared to emerging adults and adults. Nevertheless, there was no significant shape variation between all age groups $(\mathrm{p}>0.05)$ (Figure 4, Table 4).

Multivariate regression showed that only $4.12 \%$ of the time we can be confident that the shape of the MS at adolescence will be similar to the shape observed at adulthood.

\section{Discussion}

We aim to investigate the shape variation of MSamong Class III skeletal pattern in adolescents, emerging adults and adults. MS morphology is known to be influenced by facial type. Short face or forward growth of mandible has wider MS while long face or backward growth of mandible exhibits elongated and thin MS $[17,18]$. Our samples were analyzed based on its gonial angle to reduce the effect of confounding factors. In addition, it is a stable landmark to define the divergence of mandible [17] as it is part of the mandible and not influenced by other facial structures or landmarks.

The result of this study clearly showed that the MS shape in adolescents differed significantly from adults. This may be contributed by growth of craniofacial bones which does not stop after adolescence. This is in agreement with other studies where growth of craniofacial bone continues in to the third or fourth decades $[7,19]$, and bone remodelling is a continuous process throughout life $[3,4]$ This bone remodelling activity can produce differential changes in both size and shape of the bone.

Our study showed that in all the gonial angle groups, adolescents generally had a wider alveolar part, especially at the B-point area and more rounded and bulbous basal part of MS than adults. This is because adolescents experience continuous growth of mandible and MS especially during puberty. Mandibular growth accelerated significantly during adolescence. This finding is in agreement with Karlsen A T [20] who stated that the sagittal distance between Bpoint and pogonion increased from 6 to 15 years old. In addition, Rosentein 2 found that the overall AP dimension of MS increased from 8-17 years old.

Our results showed that adults in high and average gonial angle groups had significantly narrower MS, especially at the B-point and basal area compared to adolescents. The basal part is also oval shaped. This finding is supported by the study by S. Lee et al [19] where he found a continuous decrease in thickness of the alveolar bone in adults above 20 years of age. Previous study also found that different remodelling patterns were observed in different areas of MS where bone resorption of the MS mostly takes place at the labial alveolar process and the mental spine which also explain the narrowing of the B-point. Meanwhile, bone deposition occurs at the lingual side of the symphysis in both alveolar process and basal component in adolescents $(<18$ years old) and solely in the alveolar process in adults ( $>18$ years old) [4] which correlate with the narrowing of basal part in adults. In addition, the narrower MS in adults could also be explained by the slowing rate of bony turnover after reaching its peak at the age of 15-20 in women and later in men. This suggests that as age increased, there is a rapid decline in biochemical measures of bone remodelling and there is a predominance of bone resorption over bone formation [21].

We observed that the pattern of MS shape changes from adolescents to adults varied according to its vertical facial proportion. In the high gonial angle group, both adults and emerging adults have significantly narrower MS compared to adolescents. In the average gonial angle group, only the adults had significantly narrower MS, but the adolescents and emerging adults had similar shaped MS. However, it is interesting to note that in low gonial angle group, there were less distinctive MS shape differences between age groups. MS appeared to be rounded, bulbous and wider even in adults. This is in agreement with Swasty et al [22]. Who found that the cortical plate was significantly thicker on the lingual surface of the symphyseal region in subjects with low facial type. In addition, B-point area remodels differently between high and low angle groups where greater bony deposition occurs in the low angle whereas greater bone resorption occurs in the high angle group [20]. Long face has also been associated with and a resorptive nature at B-point area [20], lower biting force magnitude, reduced masticatory muscle size [23, 24], resulting in thinner MS [12].

Additionally, ageing appears to have an influence on skeletal muscle as well as its underlying bony structures, due to systemic and hormonal changes in the body [25]. Significant reduction in the cross-sectional area of the masseter and medial pterygoid muscles were observed with increasing age, from 20 to 90 years old [26]. Thus, we postulate that as age increases, high gonial angle patients 
with lesser bite force and muscular activity have a distinctively narrower MS from adolescence to adulthood. In contrast, those with low gonial angle may have maintained or slightly reduced MS dimensions due to the occlusal and masticatory forces.

Clinically, orthodontist has more room to procline or retrocline the mandibular incisors during orthodontic treatment in adolescents. Orthodontists must also be careful when moving teeth in adults with high and average gonial angle group as there is a higher risk of moving the root outside the bone which can lead to gingival recession, root resorption and bone fenestration. Decompensation of lower incisors prior to orthognathic surgery can be hazardous in the presence of thin biotype and crowding. This study also provides essential information for genioplasty, block bone grafting and implant procedures. More bone volume can be obtained from low gonial angle patients; however, cautions must be taken to prevent injury to the lower incisors as low gonial angle patients as they tend to have shorter MS. Caution should also be practiced in aged patients with high gonial angle as the alveolar part of MS is narrower. Nevertheless this pilot study concentrated on Class III skeletal base, hence comparison of MS in Class I, Class II and Class III skeletal base will be incorporated in future studies.

\section{Conclusion}

There is a noticeable pattern of shape changes in MS betweenadolescents, emerging adults, and adults.

Adults in high and average gonial angle groups had significantly narrower MS especially at the B-point and basal area compared to adolescents. Low gonial angle exhibited similar shape of MS across 3 age groups.

Mandibular symphysis shape changes are associated with increasing age.

\section{Acknowledgements}

We have no conflict of interest to disclose. The authors wish to thank Fundamental Research Grant Scheme, Ministry of Higher Education Malaysia (FRGS/1/2011/SKK/UKM/03/14) for the support in funding this project.

\section{References}

[1]. Becker MJ. Mandibular symphysis (medial suture) closure in modern Homo sapiens: preliminary evidence from archaeological populations. Am J Phys Anthropol. 1986 Apr;69(4):499-501. Pubmed PMID: 3521306.

[2]. Rosenstein SW. A longitudinal study of anteroposterior growth of the mandibular symphysis. The Angle Orthodontist. 1964 Jul;34(3):155-67.

[3]. Enlow DH, Harris DB. A study of the postnatal growth of the human mandible. American Journal of Orthodontics. 1964 Jan 1;50(1):25-50.

[4]. Martinez-Maza C, Rosas A, Nieto-Díaz M. Postnatal changes in the growth dynamics of the human face revealed from bone modelling patterns. J Anat. 2013 Sep;223(3):228-41. Pubmed PMID: 23819603.

[5]. Buschang PH, Julien K, Sachdeva R, Demirjian A. Childhood and pu- bertal growth changes of the human symphysis. Angle Orthod. 1992 Fall;62(3):203-10. Pubmed PMID: 1416240.

[6]. BJORK A. Variations in the growth pattern of the human mandible: longitudinal radiographic study by the implant method. J Dent Res. 1963 JanFeb;42(1)Pt 2:400-11. Pubmed PMID: 13971295.

[7]. Behrents RG. Growth in the ageing craniofacial skeleton. Monograph Number 17. Craniofacial Growth Series.-University of Michigan. Ann Ar bor, Michigan. 1985:238-51.

[8]. James Rohlf F, Marcus LF. A revolution morphometrics. Trends Ecol Evol. 1993 Apr;8(4):129-32. Pubmed PMID: 21236128.

[9]. Pan JY, Chou ST, Chang HP, Liu PH. Morphometric analysis of the mandible in subjects with Class III malocclusion. Kaohsiung J Med Sci. 2006 Jul;22(7):331-8. Pubmed PMID: 16849101.

[10]. Mitteroecker P, Gunz P. Advances in geometric morphometrics. Evolutionary Biology. 2009 Jun;36(2):235-47.

[11]. Artun J, Krogstad O. Periodontal status of mandibular incisors following excessive proclination. A study in adults with surgically treated mandibular prognathism. Am J Orthod Dentofacial Orthop. 1987 Mar;91(3):225-32. Pubmed PMID: 3469907.

[12]. Chung CJ, Jung S, Baik HS. Morphological characteristics of the symphyseal region in adult skeletal Class III crossbite and openbite malocclusions. Angle Orthod. 2008 Jan;78(1):38-43. Pubmed PMID: 18193946.

[13]. Izard G. The goniomandibular angle in dentofacial orthopedia. International Journal of Orthodontia, Oral Surgery and Radiography. 1927 Jul $1 ; 13(7): 578-81$.

[14]. Grubbs JB, Exline JJ, McCain J, Campbell WK, Twenge JM. Emerging adult reactions to labeling regarding age-group differences in narcissism and entitlement. PLoS One. 2019 May 15;14(5):e0215637. Pubmed PMID: 31091260.

[15]. Rohlf FJ. TpsDig, digitize landmarks and outlines, version 2.0. 2004.

[16]. Bookstein FL. Biometrics, biomathematics and the morphometric synthesis. Bull Math Biol. 1996 Mar;58(2):313-65. Pubmed PMID: 8713662.

[17]. Mangla R, Singh N, Dua V, Padmanabhan P, Khanna M. Evaluation of mandibular morphology in different facial types. Contemp Clin Dent. 2011 Jul;2(3):200-6. Pubmed PMID: 22090764.

[18]. Handelman CS. The anterior alveolus: its importance in limiting orthodontic treatment and its influence on the occurrence of iatrogenic sequelae. Angle Orthod. 1996;66(2):95-109; discussion 109-10. Pubmed PMID: 8712499 .

[19]. Lee S, Hwang S, Jang W, Choi YJ, Chung CJ, Kim KH. Assessment of lower incisor alveolar bone width using cone-beam computed tomography images in skeletal Class III adults of different vertical patterns. Korean J Orthod. 2018 Nov;48(6):349-356. Pubmed PMID: 30450327.

[20]. Karlsen AT. Craniofacial growth differences between low and high MP-SN angle males: a longitudinal study. Angle Orthod. 1995;65(5):341-50. Pubmed PMID: 8526293.

[21]. Raisz LG, Seeman E. Causes of age-related bone loss and bone fragility: an alternative view. J Bone Miner Res. 2001 Nov;16(11):1948-52. Pubmed PMID: 11697790

[22]. Swasty D, Lee J, Huang JC, Maki K, Gansky SA, Hatcher D, et al. Crosssectional human mandibular morphology as assessed in vivo by cone-beam computed tomography in patients with different vertical facial dimensions. Am J Orthod Dentofacial Orthop. 2011 Apr;139(4 Suppl):e377-89. Pubmed PMID: 21435546.

[23]. García-Morales P, Buschang PH, Throckmorton GS, English JD. Maximum bite force, muscle efficiency and mechanical advantage in children with vertical growth patterns. Eur J Orthod. 2003 Jun;25(3):265-72. Pubmed PMID: 12831216.

[24]. Proffit WR, Fields HW. Occlusal forces in normal- and long-face children. J Dent Res. 1983 May;62(5):571-4. Pubmed PMID: 6573374.

[25]. McComas AJ. Oro-facial muscles: internal structure, function and ageing. Gerodontology. 1998;15(1):3-14. Pubmed PMID: 10530166.

[26]. Newton JP, Yemm R, Abel RW, Menhinick S. Changes in human jaw muscles with age and dental state. Gerodontology. 1993 Jul;10(1):16-22. Pubmed PMID: 8300113 